\title{
Intrinsic Spin Hall Effect in Platinum: First-Principles Calculations
}

\author{
G. Y. Guo, ${ }^{1, *}$ S. Murakami, ${ }^{2}$ T.-W. Chen, ${ }^{1}$ and N. Nagaosa ${ }^{3,4}$ \\ ${ }^{1}$ Department of Physics and Center for Theoretical Sciences, National Taiwan University, Taipei 106, Taiwan \\ ${ }^{2}$ Department of Physics, Tokyo Institute of Technology, 2-12-1 Ookayama, Meguro-ku, Tokyo 152-8551, Japan \\ ${ }^{3}$ CREST, Department of Applied Physics, University of Tokyo, Tokyo 113-8656, Japan \\ ${ }^{4}$ Correlated Electron Research Center, National Institute of Advanced Industrial Science and Technology, \\ 1-1-1, Higashi, Tsukuba, Ibaraki 305-8562, Japan
}

(Received 19 April 2007; published 3 March 2008)

\begin{abstract}
Spin Hall effect (SHE) is studied with first-principles relativistic band calculations for platinum, which is one of the most important materials for metallic SHE and spintronics. We find that intrinsic spin Hall conductivity (SHC) is as large as $\sim 2000(\hbar / e)(\Omega \mathrm{cm})^{-1}$ at low temperature and decreases down to $\sim 200(\hbar / e)(\Omega \mathrm{cm})^{-1}$ at room temperature. It is due to the resonant contribution from the spin-orbit splitting of the doubly degenerated $d$ bands at high-symmetry $L$ and $X$ points near the Fermi level. By modeling these near degeneracies by an effective Hamiltonian, we show that SHC has a peak near the Fermi energy and that the vertex correction due to impurity scattering vanishes. We therefore argue that the large SHE observed experimentally in platinum is of intrinsic nature.
\end{abstract}

DOI: 10.1103/PhysRevLett.100.096401

Spin Hall effect (SHE), i.e., the transverse spin current generation by the electric field, is an issue of intensive current interests both theoretically and experimentally since the theoretical proposal for its intrinsic mechanism in semiconductors $[1,2]$. This effect enables us to control spins without magnetic field or magnetic materials, which is a crucial step for spintronics. In addition to semiconductors, the SHE in metallic systems is currently attracting interest, stimulated by experiments on the SHE or inverse spin Hall effect (ISHE), i.e., the transverse voltage drop due to the spin current [3-5]. The SHE or ISHE in metals has the following importance and advantages compared with that in semiconductors: (i) a contact with a ferromagnetic metal does not suffer from conductance mismatch [6], and one can make use of the spin-polarized current supplied from it. Thus, techniques developed in metallic spintronics can be utilized. (ii) The spin Hall conductivity (SHC) is much larger than that in semiconductors. The value of SHC obtained in Ref. [4] is 4 orders of magnitude larger than that in GaAs [7]. Naïvely this appears to be attributed to the large number of carriers, whereas the band structure is important as we discuss below. (iii) The Fermi degeneracy temperature is much higher than room temperature, and hence quantum coherence is more robust against thermal agitations than in semiconductors. We note that the spin diffusion length is relatively small in metals, e.g., $10 \mathrm{~nm}$ in platinum $(\mathrm{Pt})$ [4], causing fast decay of the SHE signal. However, it is not a crucial obstacle for observation and application, by designing the device as demonstrated in Ref. [4].

Compared with the recent experimental advances in metallic SHE, its theoretical understanding is still lacking and is urgent. Among metallic systems, Pt shows remarkably large SHE surviving even up to room temperature $[3,4]$, whereas aluminum and copper show relatively tiny $\mathrm{SHE}$ [5]. The SHC in $\mathrm{Pt}$ at room temperature is $240(\hbar / e) \times$
PACS numbers: 71.15.Rf, 72.15.Eb, 72.25.Ba, 75.47.-m

$(\Omega \mathrm{cm})^{-1}, 10$ times larger than that of aluminum at $4.2 \mathrm{~K}$. In [4] this difference is attributed to a magnitude of spinorbit coupling for each metal. However, Pt seems to be special even among heavy elements, and the SHC does not simply scale with the size of the spin-orbit coupling. Such behavior cannot be explained within the extrinsic mechanism [8-10], where material properties are represented by a few parameters such as the size of the spin-orbit coupling. This material dependence strongly suggests a crucial role of intrinsic contributions, which has been largely overlooked. It is thus highly desired to study the intrinsic SHE of Pt as a representative material for metallic SHE. This analysis opens up the possibility to theoretically design the SHE in metallic systems.

This discussion on separating intrinsic and extrinsic mechanisms is analogous to the long-standing debates on the anomalous Hall effect (AHE) [11-14]. In semiconductors, there have been experimental reports on the SHE in $n$-type GaAs [7], $p$-type GaAs [15], and $n$-type InGaN/GaN superlattices [16]. It is now recognized that the SHE in $n$-type GaAs is due to the extrinsic mechanisms, i.e., skew-scattering and side-jump contributions $[9,17]$, while that in $p$-type GaAs is mostly intrinsic $[18,19]$. In metals, the conventional understanding has been that the skew scattering is dominant in AHE. However, recent studies have revealed that the intrinsic contribution can be dominant for AHE in metals when the $\sigma_{x y}$ is of the order of $10^{3}(\Omega \mathrm{cm})^{-1}$ and the conductivity $\sigma_{x x}$ is in the range of $\sim 10^{4}-10^{6} \Omega^{-1} \mathrm{~cm}^{-1}$ [20]. This dominant contribution of intrinsic mechanism is confirmed by the detailed comparisons between the first-principles calculations [21-23] and experiments [24].

In this Letter we present an ab initio calculation for the SHC in Pt, and its analysis based on an effective Hamiltonian. We find that there are near degeneracies near the Fermi level $\left(E_{F}\right)$ at high-symmetry $X$ and $L$ points 
in the Brillouin zone (BZ) for the fcc lattice. They give a prominent enhancement of SHC in Pt. We determine an effective Hamiltonian near $X$ and $L$ points, and we demonstrate robustness of the SHE against impurities.

The band structure of $\mathrm{Pt}$ is calculated using a fully relativistic extension [25] of the all-electron linear muffin-tin orbital method [26] based on the density functional theory with local density approximation [27]. The lattice constants for Pt and $\mathrm{Al}$ used are 3.92 and $4.05 \AA$, respectively. The basis functions used are $s, p, d$, and $f$ muffin-tin orbitals for Pt but $s, p$, and $d$ muffin-tin orbitals for $\mathrm{Al}$ [26]. In the self-consistent band structure calculations, $89 k$ points in the fcc irreducible wedge (IW) of the $\mathrm{BZ}$ were used in the BZ integration. The SHC is evaluated by the Kubo formula [28]. A fine mesh of $60288 k$ points on a larger IW (3 times the fcc IW) is used. These correspond to the division of the $\Gamma X$ line into 60 segments. Comparison with test calculations with $102315 k$ points (72 divisions of the $\Gamma X$ line) for Pt indicate that the calculated SHC converges within $1 \%$.

Figure 1 shows the relativistic band structure of $\mathrm{Pt}$, and also the $\operatorname{SHC}\left(\sigma_{x y}\right)$ as a function of $E_{F}$. Remarkably, the SHC peaks at the true Fermi level $(0 \mathrm{eV})$, with a large value of $2200(\hbar / e)(\Omega \mathrm{cm})^{-1}$. This gigantic value of the SHC is orders of magnitude larger than the corresponding value in p-type semiconductors $\mathrm{Si}, \mathrm{Ge}, \mathrm{GaAs}$, and $\mathrm{AlAs}$ [28,29]. Furthermore, the calculated SHC in simple metal $\mathrm{Al}$ is only $-17(\hbar / e)(\Omega \mathrm{cm})^{-1}$, being 2 orders of magnitude smaller than that of Pt. Interestingly, the SHC in Pt decreases monotonically as the $E_{F}$ is artificially raised and becomes rather small above $3.0 \mathrm{eV}$. When the $E_{F}$ is artificially lowered, the SHC also decreases considerably, and changes its sign at $-1.1 \mathrm{eV}$. As the $E_{F}$ is further lowered, the SHC increases in magnitude again and becomes peaked at $-4.2 \mathrm{eV}$ with a large value of $-1970(\hbar / e)(\Omega \mathrm{cm})^{-1}$. The SHC decreases again when the $E_{F}$ is further lowered, and finally becomes very small below $-6.0 \mathrm{eV}$. Note that

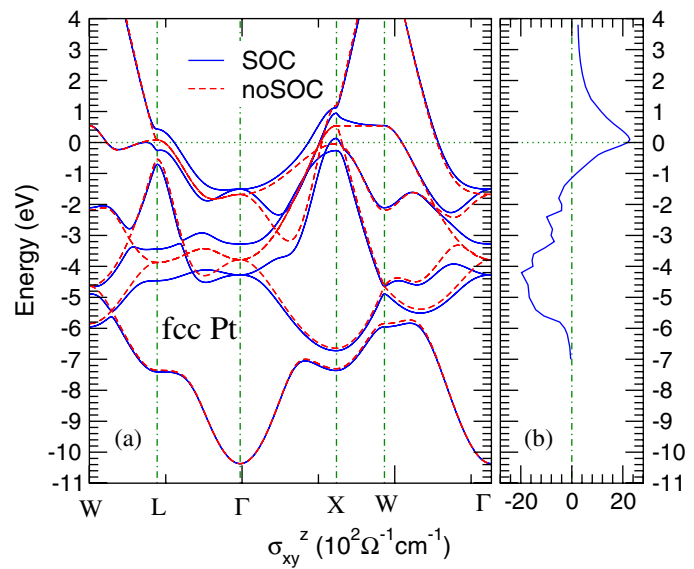

FIG. 1 (color online). (a) Relativistic band structure and (b) spin Hall conductivity of fcc Pt. The zero energy and the dotted line is the Fermi level. The dashed curves in (a) are the scalar-relativistic band structure. the bands below $-8.0 \mathrm{eV}$ and also above $2.0 \mathrm{eV}$ are predominantly of $5 s$ character and the effect of the spinorbit coupling is negligible.

We notice that a peak in the SHC appears at the double degeneracies on the $L$ and $X$ points near $E_{F}(0 \mathrm{eV})$ in the scalar-relativistic band structure (i.e., without the spinorbit coupling) while the other peak at $-4.2 \mathrm{eV}$ occurs near the double degeneracies at the $L$ and $\Gamma$ points (see Fig. 1). The double degeneracy (bands 5 and 6) at $L$ is made mostly (93\%) of $d_{x^{\prime} z^{\prime}}$ and $d_{y^{\prime} z^{\prime}}$ ( $z^{\prime}$ is the threefold axis), being consistent with the point group $D_{3 d}$ at $L$. The double degeneracy (bands 4 and 5) at $X$ consists mainly of $d_{x^{\prime} z^{\prime}}$ and $d_{y^{\prime} z^{\prime}}\left(z^{\prime}\right.$ : fourfold axis), being consistent with the point group $D_{4 h}$. These double degeneracies are lifted by the spin-orbit coupling, with large spin-orbit splittings $(\sim 0.66,0.93 \mathrm{eV}$, respectively).

One may attribute the large SHC in Pt to these double degeneracies. To see this, let us consider the $k$-resolved contribution to the SHC, i.e., Berry curvature $\Omega_{n}^{z}(\mathbf{k})$;

$$
\begin{gathered}
\sigma_{x y}^{z}=\frac{e}{\hbar} \sum_{\mathbf{k}} \Omega^{z}(\mathbf{k})=\frac{e}{\hbar} \sum_{\mathbf{k}} \sum_{n} f_{\mathbf{k} n} \Omega_{n}^{z}(\mathbf{k}), \\
\Omega_{n}^{z}(\mathbf{k})=\sum_{n^{\prime} \neq n} \frac{2 \operatorname{Im}\left[\left\langle\mathbf{k} n\left|j_{x}^{z}\right| \mathbf{k} n^{\prime}\right\rangle\left\langle\mathbf{k} n^{\prime}\left|v_{y}\right| \mathbf{k} n\right\rangle\right]}{\left(\epsilon_{\mathbf{k} n}-\epsilon_{\mathbf{k} n^{\prime}}\right)^{2}},
\end{gathered}
$$

where the spin current operator $j_{x}^{z}=\frac{1}{2}\left\{s_{z}, \mathbf{v}\right\}$, with spin $s_{z}$ given by $s_{z}=\frac{\hbar}{2} \beta \Sigma_{z}\left(\beta, \Sigma_{z}: 4 \times 4\right.$ Dirac matrices) [28]. $f_{\mathbf{k} n}$ is the Fermi distribution function for the $n$th band at $\mathbf{k}$. $\Omega_{n}^{z}$ is an analogue of the Berry curvature for the $n$th band, and it is enhanced when other bands come close in energy (i.e., near degeneracy). Figure 2(a) shows clearly that $\Omega^{z}(\mathbf{k})$ is large only near the $L$ and $X$ points. Interestingly, Berry curvature $\Omega_{n}^{z}(\mathbf{k})$ for the doublet bands 4 and 5 near the $X$ point are large but have opposite signs [Fig. 2(b)]. However, because band 5 near the $X$ point is unoccupied, only $\Omega_{n}^{z}(\mathbf{k})$ for band 4 contributes to the SHC, resulting in the large positive peak in $\Omega^{z}(\mathbf{k})$ near the $X$ point [Fig. 2(a)]. Figure 2(c) shows that the SHC decreases monotonically as the temperature $(T)$ is raised. This rather strong temperature dependence is also due to the near degeneracies since the small energy scale is relevant to the SHC there. Nevertheless, the SHC $\sigma_{x y}=240(\hbar / e) \times$ $(\Omega \mathrm{cm})^{-1}$ at $T=300 \mathrm{~K}$ is still large and is close to the measured value (240) [4]. The SHC for $\mathrm{Al}$ at 4 and $300 \mathrm{~K}$ is -17 and $-6(\hbar / e)(\Omega \mathrm{cm})^{-1}$, respectively. The former value is similar to the experimental values $(-27,-34)$ at 4.2 K [5].

In order to study the role of near degeneracies in more detail, we construct two effective Hamiltonians $H(\mathbf{k})$ for the two doubly degenerate bands at $X$ and $L$ points, respectively. At the $X$ point, by imposing the $D_{4 h}$ symmetry and the time-reversal symmetry, the effective Hamiltonian with basis $\left|\left(x^{\prime} \mp i y^{\prime}\right) z^{\prime} \uparrow\right\rangle$ and $\left|\left(x^{\prime} \pm i y^{\prime}\right) z^{\prime} \downarrow\right\rangle$ (z': fourfold axis) can be written in terms of $4 \times 4$ Clifford $\Gamma$ matrices $\left(\Gamma^{1}=\tau_{x}, \Gamma^{2}=\sigma_{z} \tau_{y}, \Gamma^{3}=\sigma_{x} \tau_{y}, \Gamma^{4}=\sigma_{y} \tau_{y}, \Gamma^{5}=\tau_{z}\right)$ as $H(\mathbf{k})=\epsilon(\mathbf{k})+\sum_{a=1}^{5} d_{a}(\mathbf{k}) \Gamma^{a}$. By expanding the coeffi- 


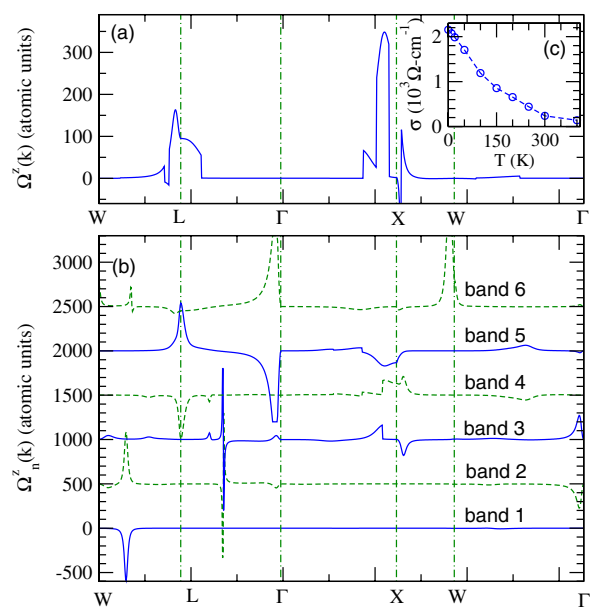

FIG. 2 (color online). (a) Berry curvature $\Omega^{z}(\mathbf{k})$ at zero temperature, and (b) band (n)-decomposed Berry curvature $\Omega_{n}^{z}(\mathbf{k})$ along the symmetry lines in the fcc Brillouin zone. In (b), $\Omega_{n}^{z}(\mathbf{k})$ for the $n$th band has been shifted upwards by $(n-1) \times 500$ for clarity. The inset (c) shows the temperature dependence of the spin Hall conductivity $\sigma_{x y}^{z}$.

cients $d_{a}$ with respect to the wave numbers $\mathbf{k}^{\prime}$ measured from $X$ and $L$ points $\left(\mathbf{k}^{\prime}=\mathbf{k}-\mathbf{k}_{i}, i=L, X\right)$, we have constructed the effective Hamiltonian. Fitting with the calculated energy bands and wave functions, we determined the expansion coefficients to $k^{14}$ order. This effective model is an even function of $\mathbf{k}^{\prime}$ and is similar to the Luttinger model, representing the valence bands of cubic semiconductors [30], or the valence and conduction bands of zero-gap cubic semiconductors [31] near the $\Gamma$ point. The previous analysis for the $p$-type semiconductors [30] are equally applied. The effective Hamiltonian has the eigenvalues $E_{l}(\mathbf{k})=\epsilon(\mathbf{k})-d(\mathbf{k})$, and $E_{u}(\mathbf{k})=$ $\epsilon(\mathbf{k})+d(\mathbf{k})$ for the lower and upper bands, respectively, where $d=\sqrt{\sum_{i=1}^{5} d_{i}^{2}}$, and these bands correspond to the heavy-hole and light-hole bands, respectively. From Eq. (35) of Ref. [30], the response of a generalized spin current (corresponding to $\Gamma^{a b}$ ) is given by

$$
\sigma_{i j}^{a b}=4 \int \frac{d \mathbf{k}}{(2 \pi)^{3}}\left(f_{\mathbf{k} l}-f_{\mathbf{k} u}\right) G_{i j}^{a b}
$$

where $f_{\mathbf{k} u}$ and $f_{\mathbf{k} l}$ are the Fermi functions of the upper and the lower bands, and $G_{i j}^{a b}=\frac{1}{4 d^{3}} \epsilon_{a b c d e} d_{c} \frac{\partial d_{d}}{\partial k_{i}} \frac{\partial d_{e}}{\partial k_{j}}$, where $\epsilon_{\text {abcde }}$ is the totally antisymmetric tensor with $\epsilon_{12345}=1$. We flipped the sign of $\sigma_{i j}^{a b}$ because the sign of the charge of the carriers is opposite from Ref. [30]. $G_{i j}^{a b}$ describes the mapping of an area form from the three-dimensional $\mathbf{k}$ space to the five-dimensional $\mathbf{d}$ space. It can be regarded as a "solid angle" enclosed by the d vector when the wave number $\mathbf{k}$ runs over the domain between the two Fermi surfaces. Hence, it becomes larger for smaller $d(\mathbf{k})=\frac{1}{2} \times$ $\left(E_{u}-E_{l}\right)$. The spin operators are given by $s^{x}=\Gamma^{35} / 2$, $s^{y}=\Gamma^{45} / 2$, and $s^{z}=\Gamma^{34} / 2$, where $\Gamma^{a b}=\frac{1}{2 i}\left[\Gamma^{a}, \Gamma^{b}\right]$. Using these relations, one can calculate the SHC $\sigma_{x y}^{z}$ from Eq. (2) by summing over the three $X$ points and four $L$ points.

The next issue is whether the contributions from various bands cancel or not. From Eq. (2), the SHC from the $X$ points and that from the $L$ points are calculated as a function of the $E_{F}$, as shown in Fig. 3. Here we put a cutoff for the $\mathbf{k}$ integral as $\pi /(5 a)$. The integrand is dominated by the contribution near the $L$ or the $X$ points, and cancellation does not occur when the Fermi energy is in the gap. It is analogous to the zero-gap semiconductors rather than GaAs [31,32]. Thus we can identify the peaks at $E_{F} \sim 0$ with the peak of the SHC in Fig. 1, and the enhancement of SHC in Pt is attributed to the near degeneracies at the $L$ and $X$ points.

As is similar to the $p$-type semiconductors [18], this intrinsic SHE is robust against impurity scattering [33]. To see this, we consider dilutely distributed short-ranged impurities $V(\mathbf{r})=\sum_{i} V \delta\left(\mathbf{r}-\mathbf{r}_{i}\right)$. It is justified in $\mathrm{Pt}$, because screening is prominent compared with semiconductors. Then the vertex corrections from the impurity scattering for the SHC vanishes in the clean limit from the following reason. Because the effective Hamiltonian satisfies (a)

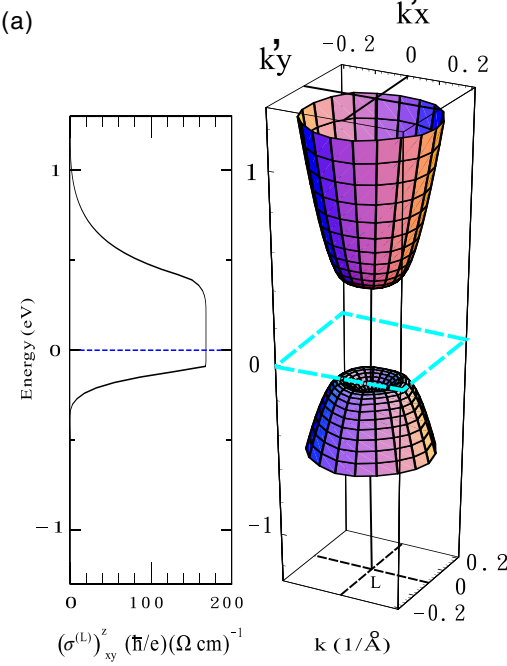

(b)

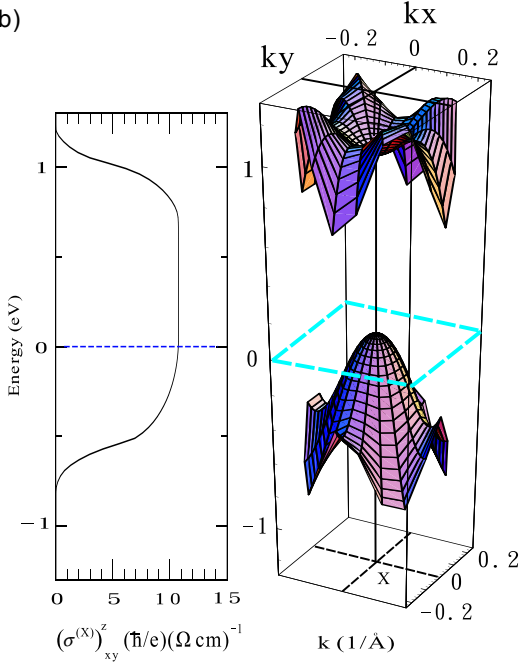

FIG. 3 (color online). Spin Hall conductivity of platinum calculated from the effective Hamiltonian for (a) the $L$ points and (b) the $X$ points, as a function of $E_{F}$ [32].

096401-3 
$H(\mathbf{k})=H(-\mathbf{k})$, the Green function is an even function and the current operator is an odd function of $\mathbf{k}^{\prime}$. Then in calculating the SHC from a correlation function between the current $j_{y}$ and the spin current $j_{x}^{z}$, the ladder diagrams from impurities cancel between the internal wave numbers $\mathbf{k}^{\prime}$ and $-\mathbf{k}^{\prime}$ for the current vertex $\mathbf{j}(\mathbf{k})$. Thus for shortranged impurities, the SHC in the clean limit is given by the intrinsic value from the bare diagram without impurity scattering. This justifies our first-principle result even in the disordered case. Although it may sound trivial, it is not in general; in the Rashba model the vertex correction from impurities is relevant and kills the intrinsic SHC even in the clean limit [17].

We note that $H\left(\mathbf{k}^{\prime}\right)=H\left(-\mathbf{k}^{\prime}\right)$ results because we restrict ourselves to the even-parity (i.e., $d$ ) orbitals. Thus even when we include the higher-order terms in $\mathbf{k}^{\prime}$ it holds true, and the vertex correction vanishes for short-ranged impurities. When $\mathbf{k}$ is away from such high-symmetry points, the orbitals with odd and even parities are hybridized, and the SHC will be canceled to some extent by the vertex corrections by impurities. Thus for inversionsymmetric systems such as $\mathrm{Pt}$, it is safe to restrict ourselves to the high-symmetry points.

Discussion on the relevance of the present result to the experiment on SHE in $\mathrm{Pt}$ [4] is in order. At room temperature the magnitude of $\sigma_{x y}^{z} \sim 240(\Omega \mathrm{cm})^{-1}$ with the conductivity $\sigma_{x x} \sim 10^{5} \Omega^{-1} \mathrm{~cm}^{-1}$ corresponds to the "intrinsic" region in the criterion of Ref. [20]. This is consistent with the idea of "resonant" Hall effect since the enhanced contribution from the near degeneracies at $X$ and $L$ points has been confirmed by the present first-principles calculation. Hence it is most probable that the SHE in Pt at room temperature is due to the intrinsic mechanism calculated in this Letter. On the other hand, at the lowest temperature the system enters the superclean extrinsic region [20], with $\sigma_{x x}$ rising up to $\sigma_{x x} \sim 10^{9} \Omega^{-1} \mathrm{~cm}^{-1}$. Hence at lowest temperature the skew scattering becomes very large, and the SHC cannot be explained only by the intrinsic mechanism.

The authors thank National Science Council and NCTS of ROC for support, and also NCHC of ROC for CPU time. The work was partly supported by Grant-inAids (Grants No. 15104006, No. 16076205, No. 17105002, No. 19740177, and No. 19019004) and NAREGI Nanoscience Project from the Ministry of Education, Culture, Sports, Science, and Technology of Japan.

Note added.-Recently, the temperature dependence of the SHC in Pt was measured to be almost constant from $T=300 \mathrm{~K}$ to $T=0 \mathrm{~K}$ [34]. Though it may look different from our scenario, it is consistent with it. In [34], the conductivity at $T=0 \mathrm{~K}$ is $\sigma_{x x} \sim 10^{5}(\Omega \mathrm{cm})^{-1}$, much lower than the above-mentioned value. This conductivity corresponds to the self-energy of the order of $10 \mathrm{meV}$, which is comparable to room temperature. This implies that the self-energy gives a cutoff to the expression of the SHC, and the SHC remains constant below room temperature. *gyguo@phys.ntu.edu.tw

[1] S. Murakami, N. Nagaosa, and S.-C. Zhang, Science 301, 1348 (2003).

[2] J. Sinova et al., Phys. Rev. Lett. 92, 126603 (2004).

[3] E. Saitoh, M. Ueda, H. Miyajima, and G. Tatara, Appl. Phys. Lett. 88, 182509 (2006).

[4] T. Kimura et al., Phys. Rev. Lett. 98, 156601 (2007).

[5] S. O. Valenzuela and M. Tinkham, Nature (London) 442, 176 (2006).

[6] G. Schmidt, D. Ferrand, L. W. Molenkamp, A. T. Filip, and B. J. van Wees, Phys. Rev. B 62, R4790 (2000).

[7] Y. Kato et al., Science 306, 1910 (2004).

[8] M. I. D’yakonov and V.I. Perel, Phys. Lett. 35, 459 (1971).

[9] H.-A. Engel, B. I. Halperin, and E. I. Rashba, Phys. Rev. Lett. 95, 166605 (2005).

[10] R. V. Shchelushkin and A. Brataas, Phys. Rev. B 71, 045123 (2005).

[11] R. Karplus and J. M. Luttinger, Phys. Rev. 95, 1154 (1954).

[12] M. Onoda and N. Nagaosa, J. Phys. Soc. Jpn. 71, 19 (2002).

[13] J. Smit, Physica (Amsterdam) 21, 877 (1955).

[14] L. Berger, Phys. Rev. B 2, 4559 (1970).

[15] J. Wunderlich et al., Phys. Rev. Lett. 94, 047204 (2005).

[16] H. J. Chang et al., Phys. Rev. Lett. 98, 136403 (2007).

[17] J.-I. Inoue, G. E. W. Bauer, and L. W. Molenkamp, Phys. Rev. B 70, 041303(R) (2004).

[18] S. Murakami, Phys. Rev. B 69, 241202(R) (2004).

[19] M. Onoda and N. Nagaosa, Phys. Rev. B 72, 081301(R) (2005).

[20] S. Onoda, N. Sugimoto, and N. Nagaosa, Phys. Rev. Lett. 97, 126602 (2006).

[21] Z. Fang et al., Science 302, 92 (2003).

[22] Y. Yao et al., Phys. Rev. Lett. 92, 037204 (2004).

[23] Y. Yao et al., Phys. Rev. B 75, 020401(R) (2007).

[24] T. Miyasato et al., Phys. Rev. Lett. 99, 086602 (2007).

[25] H. Ebert, Phys. Rev. B 38, 9390 (1988).

[26] O. K. Andersen, Phys. Rev. B 12, 3060 (1975).

[27] S. H. Vosko, L. Wilk, and M. Nusair, Can. J. Phys. 58, 1200 (1980).

[28] G. Y. Guo, Y. Yao, and Q. Niu, Phys. Rev. Lett. 94, 226601 (2005).

[29] Y. Yao and Z. Fang, Phys. Rev. Lett. 95, 156601 (2005).

[30] S. Murakami, N. Nagaosa, and S.-C. Zhang, Phys. Rev. B 69, 235206 (2004).

[31] S. Murakami, N. Nagaosa, and S.-C. Zhang, Phys. Rev. Lett. 93, 156804 (2004).

[32] As seen in Fig. 1, there is no real gap near $L$ and $X$ points while Fig. 3 shows the finite gaps. This is because we take a rather small value of the cutoff because the fitting by the second order terms in $k$ gets worse for larger cutoff. It is also the reason why the obtained value of SHC for the effective model is 1 order of magnitude smaller than the band calculation.

[33] Here we are considering an intrinsic region $10^{4}(\Omega \mathrm{cm})^{-1}<\sigma_{x x}<10^{6}(\Omega \mathrm{cm})^{-1}$, and skew-scattering contribution is neglected.

[34] L. Vila, T. Kimura, and Y. Otani, Phys. Rev. Lett. 99, 226604 (2007). 